\title{
The Use of the Voluntary Muscle Test in Leprosy Neuritis
}

\author{
C. S. GOODWIN, M.D., DIP.BACT., A.I.C.s. \\ The All-Africa Leprosy and Rehabilitation Training Centre, \\ at the Princess Zenebework Hospital, Addis Ababa, Ethiopia
}

There are 2 main problems in leprosy, the presence of leprosy bacilli and the presence of deformity. Except in the rare instances where deformity follows lepromatous infiltration of skin or bones, deformity in the limbs is secondary to involvement of peripheral nerves by the leprosy disease process. Cochrane (1964) has emphasised that unless neuritis is 'taken in hand at once, serious and crippling deformity is likely to result'. While many measures have been suggested by leprologists to reduce nerve pain, the need to assess the function of inflamed nerves and note whether treatment improves or lessens function is rarely mentioned. Criteria for assessing the results of treatment of acute ulnar neuritis in leprosy have been suggested by Harris (1964). He recommends a battery of sensory and motor tests, but for the latter prefers to measure the strength of pinch and grasp with a 'pinchometer' and a 'dynamometer', rather than to test individual muscles. However, these instruments do not distinguish between the muscles supplied by the ulnar and median nerves, and only one of these nerves may be involved at any one time in a leprosy patient. Testing the strength of individual muscles by the Voluntary Muscle Test has long been recommended for assessment of nerve function in peripheral nerve lesions (Report, 1943). If the nerves of all leprosy patients are palpated at the sites where thickening and tenderness commonly occur, a high proportion of these patients may be found to be suffering from acute or subacute neuritis. Thus a rapid, reliable and reproducible test of nerve function is required, which can be repeated serially. During the last 7 years the author has employed the
Voluntary Muscle Test, also known as the manual muscle test (Daniels et al., 1956), to assess nerve function in leprosy neuritis; and has requested physiotherapists and physiotherapy auxiliaries to perform this iest in Hong Kong, England and Ethiopia. Orthopaedic surgeons and physical medicine specialists not infrequently use the Voluntary Muscle Test to assess recovery of nerve conduction (Mannerfelt, 1968).

It is the object of this paper to show that the Voluntary Muscle Test (V.M.T.) has considerable value in the assessment of leprosy neuritis and the results of treatment. It is not suggested that the V.M.T. is a substitute for sensory training; and where sufficient staff exist many different tests of nerve function can be usefully performed, including electromyography and nerve conduction studies. Parallel electrical tests and V.M.T's were performed in one leprosy patient, and the relative value of these assessments is presented. During the last 7 years the author has continually revised the descriptions of the methods of testing each muscle, especially in the hand; and these descriptions of muscles most useful for assessment of nerve function, together with an indication of the muscles found to be most reliable are included in this paper. The descriptions and uses of V.M.T's of all hand and foot muscles will be the subject of another paper (Watson, to be published).

\section{MATERIALS}

In the Leprosy Mission leprosarium of Hay Ling Chau in Hong Kong from 1962 to 1964 , 166 patients with acute neuritis were studied and 
treated. These included men and women, and $95 \%$ were aged between 14 and 50 years of age. Voluntary Muscle Tests (V.M.T's) were done serially on all these patients at intervals varying from 3 weeks to 2 months. During 1966 and 1967, serial V.M.T's were done on 2 leprosy patients in England, one of whom was also subjected to electromyography and nerve conduction studies. In Ethiopia since September, 1967, over 200 patients with acute neuritis have been studied and treated with the aid of the V.M.T.

\section{METHODS}

Leprosy patients may draw attention to their early neuritis by complaining of nerve pain, anaesthesia or paraesthesiae, but many patients in developing countries with neuritis do not complain of nerve symptoms. One of the simplest methods for recognising early leprosy neuritis is to palpate the peripheral nerves of all leprosy patients at the sites where swelling and tenderness usually occur. The most commonly affected nerves in the arm are the ulnar at the elbow, the median just above the wrist, the radial palpable in the spiral groove, and very occasionally the median at the elbow. In the leg the lateral popliteal nerve at the head of the fibula and the posterior tibial nerve at the ankle are the most commonly affected. Any tender nerve should be rapidly assessed by the doctor or health worker for sensory and motor loss in the area supplied by that nerve; and in the absence of complete paralysis a request is made to the physiotherapist or physiotherapy auxiliary to perform a V.M.T. on the muscles supplied by each inflamed nerve.

Hands that appear normal in shape and function when examined by a casual observer may be found to be unable to perform a pulpto-pulp, thumb-to-little finger pinch; or there may be an inability to hold a piece of paper tightly between the extended ring and little fingers. When there is median neuritis, weakness of the abductor pollicis brevis may be found by a rapid test of that muscle. Patients with any of the above signs are referred for a V.M.T.
VOLUNTARY MUSCLE TEST TECHNIQUE FOR THE HAND

The Medical Research Council scale of strength of muscles (Report, 1943) is used, modified slightly in the case of strength grade 2 to a 'less than full range' rather than 'movement with gravity eliminated', as the effect of gravity in the hand is negligible:-

5. Full strength against resistance provided by the assessor's hand or finger.

4. Movement against some resistance is possible, range of movement is full.

3. There is a full range of movement, not possible against resistance (but can be done against gravity).

2. There is less than a full range of movement.

1. A flicker of movement or muscle contraction seen or felt on attempted movement, but no actual joint movement.

0 . No movement.

M.P. joints Metacarpophalangeal joints.

P.I.P. joints: Proximal interphalangeal joints.

D.I.P. joints $=$ Distal interphalangeal joints. General note: Whenever possible the strength and movement of the 2 hands are compared to detect small degrees of weakness. Italicised muscles can be accurately tested, and if these muscles alone are tested, then a reasonably accurate picture of the state of paralysis can be gauged. Resistance is always given in the direction opposite to the movement being attempted by the patient. Before applying resistance it must be first assessed whether the range of movement is full.

MUSCLES USUALLY SUPPLIED BY THE ULNAR NERVE

Flexor carpi ulnaris (F.C.U.). With the palm up, the patient flexes the wrist with ulnar deviation. The assessor feels for the muscle tendon just proximal to the pisiform bone.

Flexor digitorum profundus (F.D.P.) to the ring and little fingers. With the patient's palm up, the assessor maintains extension of the M.P. 
and P.I.P. joints of the finger being tested, and all joints of the other fingers. The patient then flexes each D.I.P. joint. Resistance is applied by pressure to the pulp of each finger in turn.

Abductor digiti minimum (A.D.M.). With the palm up, the little finger is flexed slightly at the M.P. joint and abducted, keeping the other joints extended. Resistance is applied by pressing the little finger towards the fingeradducted position.

Opponens digiti minimi (O.D.M.). With the palm up, the little finger is flexed slightly at the M.P. joint and deviated radially keeping the finger straight.

lst dorsal interosseous (D.Int.1). With the ulnar border of the hand on the table, the fingers extended and the thumb relaxed, the index finger is abducted. Watch for muscle bulk which is obvious on contraction. For resistance-press on the proximal phalanx of the index finger, pushing finger towards adducted position.

Lumbricals 3 and 4 (Lumb.)-ring and little fingers. With the dorsal surface of the hand upwards and the wrist slightly extended, while keeping the M.P. joints at $90^{\circ}$, the D.I.P. and P.I.P. joints are straightened. Resistance is applied by pressure on the palmar side of each proximal phalanx.

MUSClES USUALIY SUPPLIED BY MEDIAN NERVE

Pronator teres (Pro.T.). With the elbow at $90^{\circ}$, the arm adducted, and the palm facing upwards, the forearm is pronated, i.e., rotated inwards, without moving the elbow, until the palm is facing downwards.

Flexor carpi radialis (F.C.R.) and palmaris longus. With the palm up, the wrist is flexed and the combined strength of the two muscles assessed. Watch for the tendons near to the middle of the front of the wrist. Palmaris longus muscle is sometimes absent.

Flexor digitorum sublimis (F.D.S.) to index, long, ring and little fingers. With the back of the hand on the table, hold down the fingers not being tested. The patient then bends the finger being tested at the P.I.P. joint, keeping M.P. and D.I.P. joints straight. For resistancepress down on the anterior aspect of the middle phalanx. Watch for substitution by the flexor digitorum profundus, by making sure that the D.I.P. joint stays relaxed.

Flexor pollicis longus (F.P.L.). The assessor holds the thumb M.P. joint extended and the patient flexes the I.P. joint. For resistancepress upon the distal phalanx of the thumb.

Flexor digitorum profundus (F.D.P.) to the index and long fingers. See F.D.P. for the ring and little fingers.

Abductor pollicis brevis (Ab.P.B.). With the hand on the table, palm up and the thumb on the index finger, the patient abducts the thumb straight up from the index finger and at right angles to the palm. For resistance the assessor presses the thumb straight down towards the index finger.

Opponens pollicis (Opp.P.). The patient abducts the thumb as described for the abductor pollicis brevis, and then brings it across the palm towards the little finger, keeping the thumb I.P. and the M.P. joints extended. Resist in an opposite direction, pressing at the M.P. joint.

Lumbricals 1 and 2-the index and long fingers. (See Lumbricals for ring and little fingers.)

MUSCLES SUPPLIED BY THE RADIAL NERVE

Brachio-radialis (Br.Rad.). With the elbow at $90^{\circ}$, the arm abducted and the thumb up, the patient flexes the elbow. For resistance-the assessor presses down at the wrist.

Extensor carpi radialis longus and brevis (E.C.R.L. and B.). With the hand on the table, pa'm down and the fingers flexed, the patient extends the wrist and deviates it radially. The muscle tendons can be seen together on the radial side of the dorsum of the wrist.

Extensor carpi ulnaris (E.C.U.). Test in a similar way to E.C.R.L. and B., but the wrist is dorsiflexed in ulnar deviation.

Extensor digitorum (E.D.). With the fingers extended the M.P. joints are extended. For resistance-the assessor presses down on the proximal phalanges. 
Extensor pollicis longus (E.P.L.). With the hand resting on the ulnar side of the little finger, the thumb relaxed, the assessor holds the thumb M.P. joint, and the patient extends the distal joint. For resistance-the assessor presses down on the distal phalanx.

V.M.T. TECHNIQUE FOR MUSCLES SUPPLIED BY THE LATERAL POPLITEAL NERVE

Tibialis anterior (Tib.A.). With all the toes relaxed or actively flexed, the patient inverts and dorsiflexes the foot. The tendon can be seen and felt. Resist in the opposite direction.

Extensor digitorum longus (E.D.L.). Holding the ankle at $90^{\circ}$, the patient extends the lateral 4 toes. The 4 tendons to the toes can be seen and felt. Resist by pressure over the proximal phalanx of each toe in turn.

Extensor hallucis longus (E.H.L.). Holding the ankle at $90^{\circ}$, the patient extends the great toe. Resist by pressing against the proximal phalanx.

Peronei (Per.). Patient plantarflexes and everts the foot. Tendons of $\mathrm{P}$. longus and brevis can be seen and felt. Resist by pushing against length of 5th metatarsal. These muscles can be tested together but can be distinguished from each other if necessary by watching for depression of the lst metatarsal which indicates action of the longus muscle.

\section{RESULTS}

From November, 1962, to June, 1964, of the 166 Chinese patients with early neuritis all but 2 showed improvement in their serial V.M.T's. In the few patients whose V.M.T's initially showed that some muscles were becoming weaker, it was usually found that the limb was not being rested in a sling; but such an activity as digging in their garden was being indulged in. More extensive drug therapy was instituted and some patients were admitted to hospital bed rest. When nerve pain was uncontrolled by rest and drug therapy, and the V.M.T. showed that some nerve function was still present, operations were performed to relieve pressure on the affected nerve. A few case histories may illustrate the value of V.M.T's:-
1. A.B. Chinese male, 25, hospital number 1590. On admission to the Hong Kong Leprosarium in August, 1963 , he was found to have a left low ulnar paralysis, and early median and radial weakness (Table 1).

2. S.K. Chinese female, aged 40, hospital number 1573. Admitted 20th July, 1963, with acute exacerbation of borderline leprosy. She had bilateral ulnar and median weakness and a right drop foot. She was put to bed and cotton wool was bandaged round both elbows, bilateral wrist splints were bandaged on, and a plaster of Paris back slab was made for the right leg to keep the ankle at $90^{\circ}$. Prednisolone was given in a full course. A V.T.M. on the 24th July revealed that there was almost a complete paralysis of the muscles supplied by the right lateral popliteal nerve. However, 6 weeks later the tibialis anterior muscle was strength 4 , and the extensor hallucis, extensor digitorum and peronei were half strength (Table 2). On 4th November, 1963, more recovery was found and by 13th February, 1964, all the previously paralysed muscles were at full strength.

3. Mrs. E.F., female, aged 50. In England in November, 1966 , this woman with leprosy was found to have a tender right ulnar nerve and both median nerves were tender. She had an established left ulnar paralysis. A V.T.M. was performed which indicated a low ulnar neuritis on the right (Table 3), and bilateral median weakness (Table 4). On the 7 th December, 1966, an electrodiagnostic examination was performed by Dr. K. H. Nixon, consultant in physical medicine. The following is taken from his report:-

(i) Nerve excitability studies: These show a decrease in the excitability in the left ulnar nerve.

(ii) Motor nerve conduction velocity studies: These show a delay in the right median nerve of $10 \mathrm{~m} /$ secs from wrist to abductor digiti minimi.

(iii) Sensory nerve conduction velocity studies: In the right hand from wrist to little finger there is a good sensory response at $7 \mathrm{~m} / \mathrm{secs}$, the upper limit of normal.

(iv) I /T Curves: Partial denervation is demonstrated in the median nerve territory on both sides (Table 5) and in the ulnar nerve territory on the right. There is no denervation in the flexor carpi ulnaris on the right; denervation is in the nerve distal to the nerve origin of this muscle.

(v) Electromyography: This was performed on the abductor pollicis brevis, abductor digiti minimi on the right, and the flexor carpi ulnaris. These show the presence of fibrillation potentials in the abductor pollicis brevis on the right. Interference pattern was complete in the abductor pollicis brevis and reduced in the abductor digiti minimi on the right, and discrete in the second dorsal interosseous on the left.

Note: If the I/T curves are plotted graphically it can be seen that a greater degree of denervation has occurred on the left than on the right, and the V.T.M's reflect this finding.

The electromyography needles caused agonising pain to this patient and she was quite unwilling to submit to a second electrodiagnostic examination. However, 
TABle 1

A.B. Chinese male, aged 25. V.M.T. of left hand. August, 1963

\begin{tabular}{lrlrlr}
\hline \multicolumn{2}{c}{ Ulnar-(Muscles $)$} & \multicolumn{2}{c}{ Median } & \multicolumn{2}{c}{ Radial } \\
\hline F.C.U. & 5 & Pro. T. & 5 & Br. Rad. & 5 \\
F.D.P. 3, 4 & 4,3 & F.C.R. & 5 & E.C.R.L. and B. & 5 \\
A.D.M. & 0 & Palm L. & 5 & E.D. & 4 \\
O.D.M. & 0 & F.D.S. 1-4 5554 & E.C.U. & 5 \\
D. Int. fig. I. & 0 & F.P.L. & 5 & E.P.L. & 4 \\
Lumb. 3 and 4 & 0,0 & F.D.P. 1, 2 5, 5 & & \\
& & Ab.P.B. & 4 & \\
& & Opp.P. & 5 & \\
\end{tabular}

TABLE 2

S.K. Chinese female, aged 40. Right foot V.M.T.

\begin{tabular}{lcccc}
\hline & 24.7 .63 & 3.9 .63 & 4.11 .63 & 13.2 .64 \\
\hline Tibialis anterior & 0 & 4 & 5 & 5 \\
Extensor hallusis & 0 & 2 & 3 & 5 \\
Extensor digitorum & 1 & 3 & 4 & 5 \\
Peronei & & 3 & 5 & 5 \\
\hline Passive dorsiflexion & $75^{\circ}$ & $70^{\circ}$ & $70^{\circ}$ & $70^{\circ}$ \\
Active dorsiflexion & $105^{\circ}$ & $87^{\circ}$ & $80^{\circ}$ & $80^{\circ}$ \\
\hline
\end{tabular}

TABLE 3

Mrs. E.F., aged 50. Right hand.

V.M.T's ulnar-supplied muscles

\begin{tabular}{lccc}
\hline & 14.12 .66 & 8.2 .67 & 19.4 .67 \\
\hline F.C.U. & 4 & 5 & 5 \\
F.D.P. & 4 & 4 & 5 \\
A.D.M. & 4 & 4 & 4 \\
D.Int.I. & 3 & 4 & 4 \\
Lumb. 3, 4 & 4 & 4 & 5 \\
\hline
\end{tabular}

TABLE 4

Mrs. E.F. V.M.T's of median supplied musclesleft is weaker than right

\begin{tabular}{cclcc}
\hline \multicolumn{2}{c}{ RIGHT } & & \multicolumn{2}{c}{ LEFT } \\
19.4 .67 & 14.12 .66 & & 14.12 .66 & 19.4 .67 \\
\hline 5 & 5 & Pro.T. & 4 & 4 \\
5 & 4 & F.C.R. & 4 & 4 \\
5 & 5 & F.D.S., 1-4 & 5 & 5 \\
4 & 4 & F.D.P. 1, 2 & 4 & 4 \\
5 & 4 & An.P.B. & 3 & 3 \\
5 & 4 & Opp.P. & 4 & 4 \\
5 & 4 & Lumb. 1, 2 & 4 & 4 \\
\hline
\end{tabular}

TABLE 5

Mrs. E. F. Part of electrodiagnostic examination. December, 1966

\begin{tabular}{crcc}
\hline$I / T$ Curves & & Right & Left \\
& & & \\
Abductor pollicis brevis & $100 \mathrm{~m} / \mathrm{s}$ & & 75 \\
$30 \mathrm{~m} / \mathrm{s}$ & 1 & 8 \\
$10 \mathrm{~m} / \mathrm{s}$ & 1.3 & 1.6 \\
$3 \mathrm{~m} / \mathrm{s}$ & 2.3 & 5.0 \\
$1 \mathrm{~m} / \mathrm{s}$ & 4.1 & 6.0
\end{tabular}

TABLE 6

T.A. Ethiopian male, aged 18. Serial V.M.T's of ulnar-supplied muscles

\begin{tabular}{ccccccc}
\hline $\begin{array}{c}\text { RIGHT } \\
8.11 .67\end{array}$ & & & \multicolumn{1}{c}{ LEFT } \\
21.67 & 3.1 .68 & 21.2 .68 & 4.4 .68 & 7.5 .68 \\
\hline 5 & F.C.U. & 5 & 5 & 5 & 5 & 5 \\
5555 & F.D.P. & 5554 & 5554 & 5554 & 4545 & 4545 \\
5 & A.D.M. & 3 & 3 & 5 & 3 & 5 \\
5 & O.D.M. & 0 & 2 & 5 & 3 & 4 \\
5 & D.Int.1 & 3 & 4 & 4 & 5 & 5 \\
5 & 2 & 0 & 3 & 4 & 5 & 5 \\
5 & 3 & 0 & 2 & 2 & 4 & 5 \\
5 & 4 & 0 & 2 & 2 & 3 & 4 \\
5 & Lumb. 4 & 0 & 2 & 2 & 2 & 3 \\
5 & 3 & 2 & 2 & 3 & 3 & 3 \\
\hline
\end{tabular}


she was taught hand exercises for her weak muscles, given a sling for the right arm and appropriate drug treatment. Serial V.T.M's were performed and showed recovery of muscle strength after 4 months (Tables, 3 and 4).

4. T.A. Ethiopian male, aged 18. This young man was seen in November, 1967, when his B.I. was 0.0, after he had been treated for 2 years for borderline leprosy. He complained of pain in his ulnar nerves which were found to be grossly enlarged and tender, and the left hand had demonstrable ulnar weakness. A V.T.M. showed that the right hand ulnarsupplied muscles were normal strength, while the left hand had a low ulnar neuritis with marked weakness of most of the short muscles of the hand (Table $6)$. He was admitted to hospital and treated with a full course of Prednisolone. A V.T.M. on 3rd January, 1968, showed recovery in nearly all the muscles, with more improvement by the 21 st February. He was then treated as an out-patient, but in April he complained again of acute nerve pain and a V.T.M. showed that there was slight weakness of some muscles which had been at normal strength in February (Table 6). This was regarded as an indication for hospital admission, and he was also treated with Prednisolone again. AV.T.M. on the 7th May, 1968, showed recovery of strength, and presumably of nerve function in nearly all the muscles. He was discharged again for out-patient treatment.

\section{DISCUSSION}

To assess the presence and extent of nerve damage in leprosy neuritis it has been shown that the Voluntary Muscle Test (V.M.T.) is extremely valuable. It is doubtful whether complex electrodiagnostic studies add much useful information to that obtained by a V.M.T. except in very early neuritis. Inasmuch as a V.M.T. of the muscles in one hand relevant to reveal neuritis can be efficiently performed in 5 or 10 minutes and involves no equipment, it is suggested that, for the time required, this test provides a gieater amourit of information than can be acquired by any other test; and should be recorded for every patient found to have early neuritis. Sensory testing requires a much greater length of time for one test, and serial sensory tests require many record sheets, in contrast to the V.M.T. which is recorded in parallel columns; and with the latter test improvement or worsening can be seen at a glance. A comparison of V.M.T. results and the stage at which protective sensation is retained in the hand will be the subject of a further paper. However, it can be stated that among Chinese patients, and in the few Ethiopian patients so far studied, definite weakness of the short muscles of the hand occurs in most patients before loss of protective sensation. Thus the V.M.T. can be used not only to recognise the stage at which normal hand movements can be saved, but also the stage at which protective sensation probably can be saved; and as such can be seen to be of double value.

Mendis (1965) in his booklet on physiotherapy in leprosy devotes a very small paragraph to the problem of neuritis. He advocates the use of electrical assessments, namely 'intensityduration graphs', and mentions that 'these graphs detect minor degrees of denervation that may escape a clinical assessment'. However, the equipment and skill required for successfully making these electrical graphs are rarely found in leprosy centres and the time required renders them impracticable in circumstances where large numbers of leprosy patients are being treated and the great majority of patients with early neuritis are being detected. The Manual muscle test has been used by Furness (1967) to assess function during treatment for recent paralysis in leprosy patients, and at the National Institute of Allergy and Infectious Diseases in Maryland (Atkinson et al., 1967). The M.R.C. muscle tests (Report, 1943) are also used by Parry (1958).

In the 'Manual for training leprosy rehabilitation workers' (Itch and Eason, undated), the strength of muscles is classified as 'functional' or 'non-functional'. However, small degrees of improvement or regression are difficult to assess when these 2 grades alone are used, and this classification would divorce leprosy physiotherapy from the main-stream of physiotherapy in the world.

Harris (1964) has pointed out that claims for the successful treatment of leprosy neuritis have been worded extremely vaguely, 'excellent', 'beneficial', 'successful'. He recommends that many sensory tests should be performed, including the ninhydrin test for sweating. He couples these delicate tests with observations which would appear to be less delicate, such as wasting, and the patient's type of grasp, whether

\section{Leprosy Review}


the fingertips dig into the forearm of the examiner; but both of these tests are difficult to record objectively. He describes measurement of the strength of pinch and grasp with a 'pinchometer' and a 'dynamometer'. The dynamometer is composed of a rubber bulb and an air pressure gauge filled with hydraulic oil and then air. However, in leprosy hospitals in developing countries this instrument can easily become damaged and inefficient (Ward, D. J., personal communication).

The 'fatigue' test for early ulnar paralysis is obviously of great value (Harris, 1964), but if very delicate tests are used routinely, treatment must be instituted correspondingly early and the number of patients under treatment will be vastly increased, probably beyond the bounds of possibility that one unit could manage at any one time. Furthermore the patient may be unco-operative under treatment if he does not feel that his hand is abnormal. Harris acknowledges that some degree of spontaneous recovery occurs in many forms of neuritis, and thus it would be reasonable to treat as a first priority patients whose neuritis is more than minimal.

Watson (1965) has described her opinion of the V.M.T. She states that she has found the V.M.T. 'completely satisfactory for routine testing, and would recommend it for use in most institutions. The tests can be taught to most technicians who have learned the positions of the muscles of the hand and forearm. They do need to be carefully taught; and accuracy is acquired only by care, by practice, and by knowledge of trick movements to be avoided. My 3 patient assistants, none of whom have completed their 'middle-school' education, have all learned the position of the hand muscles, by way of diagrams and explanations, and are all doing voluntary muscle tests routinely.'

\section{SUMMARY}

The Voluntary Muscle Test (V.M.T.) is a rapid, reliable and reproducible assessment of the extent of nerve damage in leprosy neuritis, and requires no equipment. Definite muscle weakness is revealed in hands that appear normal to a casual observer, and in such patients active treatment for neuritis is immediately indicated. The results of treatment can be objectively judged by serial V.M.T's, and when V.M.T's are recorded in parallel columns they can be rapidly compared. For speed, interpretation and comparability V.M.T's are superior to sensory tests. As a guide to the treatment of patients in developing countries, electrical studies probably do not add much vital injormation to that obtained by a V.M.T. Restoration of nerve function and muscle strength as shown by serial V.M.T's may frequently be accompanied by a preservation of protective sensation in the hand. The technique of doing a V.M.T. can be readily taught to nurses and physiotherapy auxiliaries, and has been found to be invaluable and entirely satisfactory in Chinese, English and Ethiopian leprosy patients.

\section{ACKNOWLEDGEMENTS}

The author is happy to acknowledge his debt to Miss Jean Watson, M.c.s.P., Mr. David Ward, M.C.s.P., and Mrs. Marguerite Engardt, leprosy physiotherapists, without whom this paper could not have been written. Dr. K. H. Nixon, consultant to the Portsmouth group of hospitals, is thanked for his permission to quote from his report on patient E.F.

\section{REFERENCES}

1. Atkinson, A. J., Sheagren, J. N., Rubio, J. B. and KNIGHT, v. (1967). Evaluation of B663 in human leprosy. Int. J. Lepr., 35, 119.

2. DANIELS, LUCILlE, WILliAMS, MARIAN and Worthingham, catherine (1956). Muscle Testing, 2nd Ed. Philadelphia: Saunders. p. 10.

3. Cochrane, R. G. (1964). Neuritis in leprosy. In Leprosy in theory and practice, 2nd edition. Ed. by Cochrane, R. G. and Davey, T. F. Bristol: John Wright, p. 412.

4. FUrness, M. A. (1967). Physical therapy in the management of recent paralysis in leprosy. Lep. Rev., 38, 193.

5. HARRIS, J. (1964). Criteria for assessing the results of treatment of acute ulnar neuritis in leprosy. Lep. in India, 36, 107.

6. Iтсн, M. and EAson, ALice L. (undated). Manual for training leprosy rehabilitation workers. New York: Internat. Soc. for Rehabilitation of the Disabled. 
7. MANNERFELt, L. (1968). Median nerve entrapment after dislocation of the elbow. J. Bone and Joint Surg., 50B, 152.

8. mendis, м. (1965). Physiotherapy in leprosy. Bristol: John Wright, p. 8.

9. PARRY, C. B. w. (1958). Rehabilitation of the hand. London: Butterworth, pp. 71 and 93.
10. REPORT (1943). Aids to the investigation of peripheral nerve injuries. Med. Res. Council War Memo, No. 7. 2nd Edition. London: H.M. Stationery Office.

11. Watson, JeAN M. (1965). In Papers on leprosy rehabilitation given at the Third Pan-Pacific Rehabilitation Conference, Tokyo, 1965. New York: International Soc. for Rehabilitation of the Disabled, p. 22. 